\title{
20-kHz alternating current stimulation: effects on motor and somatosensory thresholds
}

\author{
Diego Serrano-Muñoz ${ }^{1,2}$, Juan Avendaño-Coy ${ }^{2^{*}}$ (D), Cristina Simón-Martínez ${ }^{3}$, Julian Taylor ${ }^{1}$ and \\ Julio Gómez-Soriano²
}

\begin{abstract}
Background: High frequency alternating current (HFAC) stimulation have been shown to produce a peripheral nerve conduction block. Currently, all the studies applying HFAC stimulation in clinical studies, have employed frequencies below $10 \mathrm{kHz}$. The main aim of this work was to investigate the neuromodulatory effect of transcutaneous $20 \mathrm{kHz}$ stimulation on somatosensory and pain thresholds, and maximal handgrip strength.
\end{abstract}

Methods: A randomized, crossover, single-blinded, placebo-controlled trial was conducted following recruitment of fourteen healthy volunteers. Transcutaneous stimulation at $20 \mathrm{kHz}$ and sham stimulation were applied over the ulnar and median nerves of fourteen healthy volunteers for $20 \mathrm{~min}$. Maximal handgrip strength (MHS), mechanical detection threshold (MDT) and pressure pain threshold (PPT) were registered prior to, during (15 min), immediately after the end (20 min), and 10 min following stimulation.

Results: The $20 \mathrm{kHz}$ stimulation showed a lower MHS during the stimulation at the $15 \mathrm{~min}$ (30.1 kgs SE 2.8) and at 20 min (31.8 kgs, SE 2.8) when compared to sham stimulation (35.1 kgs, SE 3.4; $p<0.001$ and $34.2 \mathrm{kgs}, \mathrm{SE} 3.4 ; p=$ 0.03 , respectively). The $20 \mathrm{kHz}$ stimulation resulted in a slight increase in MDT at $15 \mathrm{~min}(0.25 \mathrm{mN} ; 0.25-2.00)$ when compared to the sham stimulation $(0.25 \mathrm{mN} ; 0.25-0.25 ; p=0.02)$, and no effects were showed for PPT.

Conclusions: High-frequency stimulation at $20 \mathrm{kHz}$ suggests a partial block of nerve activity. Studies in subjects with neurological disorders characterized by nerve hyperactivity are needed to confirm the clinical impact of this non-invasive electrical stimulation technique.

Trial registration: NCT, NCT02837458. Registered on 12 April 2017.

Keywords: Nerve block, High-frequency, Transcutaneous electric stimulation, Peripheral nerve

\section{Background}

Many neurological pathologies lead to neuromuscular dysfunction characterized by an abnormal increase in neural activity, such as the spasticity syndrome or chronic evoked pain [1]. Exaggerated neuromuscular activity requires therapeutic treatment, which may be based on non-pharmaceutical strategies, including neuromodulation using electrical stimulation to reduce neuronal hyperexcitability. High-frequency alternating current (HFAC) stimulation applied at frequencies

\footnotetext{
* Correspondence: juan.avendano@uclm.es

${ }^{2}$ Toledo Physiotherapy Research Group (GIFTO), Faculty of Physiotherapy and Nursery, Castilla La Mancha University, 45071 Toledo, Spain

Full list of author information is available at the end of the article
}

higher than $1 \mathrm{kHz}$ have been shown to produce a peripheral nerve conduction block in preclinical studies, an effect which is rapidly reversed after stimulation [2]. Studies using experimental models have demonstrated that the minimum stimulation frequency required to produce a conduction block without causing damage to the peripheral nerve is approximately within the range of $4-5 \mathrm{kHz}$ [3-5]. Although HFAC has been shown to be a safe technique, only a few studies using this stimulation protocol has been applied to humans [6-11]. The application of HFAC stimulation using implanted devices reveal positive therapeutic effects with regards to reducing body weight following the intermittent conduction block of vagal nerve activity in obese people $[8,12]$,

(c) The Author(s). 2020 Open Access This article is distributed under the terms of the Creative Commons Attribution 4.0 International License (http://creativecommons.org/licenses/by/4.0/), which permits unrestricted use, distribution, and 
in reducing tinnitus after auditory nerve block with a HFAC applied at $5 \mathrm{kHz}$ [13], and also in reducing pain using $10 \mathrm{kHz}$ following limb amputation [9].

To date only four published studies have reported the effects of non-invasive transcutaneous HFAC stimulation over peripheral nerves $[7,10,11,14]$. In a previous study conducted by our group, $5 \mathrm{kHz}$ HFAC stimulation applied over the radial nerve for 20 min produced similar modulatory effects on somatosensory thresholds when compared to transcutaneous electrical nerve stimulation (TENS) [7]. Another pilot study demonstrated a larger decrease in maximal handgrip strength during $10 \mathrm{kHz}$ HFAC of the median and ulnar nerve, when compared to either a $5 \mathrm{kHz}$ or sham stimulation [14]. Similar results have been shown by Kim et al. [10], reported an increase in mechanical detection threshold (MDT) and pressure pain threshold (PPT) after $10 \mathrm{kHz}$ HFAC stimulation of the median nerve, and an additional decrease in finger muscle contraction during the stimulation. Finally, Springer et al. [11], who showed that the application of $7 \mathrm{kHz}$ HFAC to the ulnar nerve resulted in a reduction in muscle activity of the abductor digiti minimi, as measured with electromyography, immediately after HFAC stimulation.

Altogether, these clinical experimental studies support the potential effect of HFAC stimulation on nerve conduction block, even though the optimal stimulation frequency remains unknown. Currently, all the studies applying HFAC stimulation in clinical studies [15], using either invasive electrodes or transcutaneous techniques, have employed frequencies below $10 \mathrm{kHz}$. However, a study performed by Ackermann et al. [16] in nonhuman primates, whose nerve diameter is similar to humans, showed that the most effective stimulation frequency was in the range of $20-40 \mathrm{kHz}$, with the 10 $\mathrm{kHz}$ stimulation protocol being ineffective for nerve conduction block. More animal studies have observed that frequencies higher than $10 \mathrm{kHz}$ was more effective for obtaining partial nerve block [17-19].

To date no clinical study has employed transcutaneous HFAC at frequencies greater than $10 \mathrm{kHz}$. Therefore, the main objective of this study was to investigate the neuromodulatory effect of transcutaneous HFAC stimulation applied on the median and ulnar nerve at a frequency of $20 \mathrm{kHz}$ on somatosensory and pain thresholds and maximal handgrip strength in healthy participants, compared to sham stimulation.

\section{Methods}

\section{Design}

A randomized, crossover, single-blinded, placebocontrolled trial was conducted following recruitment of fourteen healthy volunteers. All volunteers signed the informed consent form approved by the Local Ethics
Committee (Ref. No. 158; 02/11/2017). The present study was registered in the ClinicalTrials.gov Protocol Registration System (NCT02837458). Participants received both interventions $(20 \mathrm{kHz}$ and sham stimulation), and the order of these interventions was randomized using a web page tool (www.randomizer.org). The duration of the active or sham intervention was $20 \mathrm{~min}$. The evaluation included measures A, B and C (see section $D$. Outcome measures) assessed throughout the experiment at four time points: i) before stimulation (0 $\mathrm{min})$, ii) during stimulation at $15 \mathrm{~min}(15 \mathrm{~min})$, iii) at the end of the stimulation $(20 \mathrm{~min})$ and iv), at $10 \mathrm{~min}$ after the end of the stimulation $(30 \mathrm{~min})$. A washout period of $24 \mathrm{~h}$ was used between the application of the two interventions [14].

\section{Subjects}

Healthy volunteers between 18 and 65 years old, without any peripheral or central nervous system pathology, were recruited using non-probabilistic convenience sampling. The exclusion criteria included musculoskeletal pathology in the upper limb, inability to tolerate electrical current stimulation, allergy to the stimulating electrode material, pacemaker or any other implanted device, epilepsy, neurotrauma, recent surgical procedures, diabetes, pregnancy, and cancer.

\section{Intervention}

Subjects were seated with their dominant elbow flexed at $90^{\circ}$. Two surface self-adhesive electrodes $5 \mathrm{~cm} \times 5 \mathrm{~cm}$ (ValuTrode, Axelgaard Manufacturing Co, LTD, Fallbrook, USA) were used. The proximal electrode was fixed to the skin over the path of the ulnar nerve at the epitrochlea and the distal electrode was placed on the median nerve over the carpal tunnel [14]. The same stimulator device was used to apply the two interventions (Myomed 932, Enraf-Nonius, Delft, Netherlands), whose software was previously modified to be able to deliver an electrical current of $20 \mathrm{kHz}$. Interferential tetrapolar mode was used, although only two electrodes (Channel 1 ) was connected. The other two electrodes (Channel 2) were not used to avoid any affect due to interference. A digital oscilloscope (Tektronix TDS2014B, Beaverton, USA) was used to confirm that the pulse was biphasic sinusoidal waveform with no modulation and with a frequency of 20 $\mathrm{kHz}$.

\section{$20 \mathrm{kHz}$ stimulation}

A charge-balanced, symmetric, biphasic sinusoidal current without modulation was presented at a frequency of $20 \mathrm{kHz}$ for the active intervention. The stimulation intensity was defined as that sufficient to produce a "strong but comfortable" sensation, just below motor threshold [20]. The intensity was gradually increased 
until a minimal visible contraction was observed and then subsequently decreased until the muscle contraction disappeared, with the same intensity maintained throughout the stimulation. To avoid habituation to the stimulus, participants were asked to corroborate the perceived stimulation sensation every $2 \mathrm{~min}$, so that the stimulation intensity could be increased if requested [21, 22].

\section{Sham stimulation}

Sham stimulation was applied with the same device and electrode placement, but the intensity was adjusted by simulating to progressively increase the intensity of a non-connected channel. Participants were also blinded to the hypothesis of the study by being informed that in some cases the perceived sensation might be of different as the stimulus intensity could be adjusted to below their sensory threshold, with the possibility that the participant may or may not feel the stimulus current $[7,20]$.

\section{Outcome measures}

\section{Maximal handgrip strength (MHS)}

Maximal voluntary handgrip muscle strength was measured using a handgrip dynamometer (Grip Strength Dynamometer T.K.K. 5401 GRIP-D Takei Scientific Instruments CO., LTD. Shinagawa-ku, Tokyo). Average maximal handgrip muscle strength was calculated as mean of three measurements and used for statistical analysis [14] (Fig. 1a). The MHS outcome was reported in kilograms.

\section{Mechanical detection threshold (MDT)}

Mechanical sensory threshold was examined at the palmar surface of the hand, over a $1 \mathrm{~cm}^{2}$ surface area at the hypothenar eminence. The MDT was assessed using modified Von Frey filaments (OptiHair2, MARSTOCKnervtest, Marburg, Germany), using the following calibrated filaments to apply forces of $0.25,0.5,2,4,8$, 16, 32, 128, 256 y $512 \mathrm{mN}$ [23]. Participants were instructed to close their eyes during the sensory examination and say 'yes' when perceived the sensory stimulus. The sensory examination involved seven applications for each filament. MDT was defined when sensory perception was successful for at least 4 of the 7 times (Fig. 1b).

\section{Pressure pain threshold (PPT)}

The PPT was assessed with the application of a digital algometer (Wagner Instruments, FORCE ONE model FDIX, USA) applied on the triquetral bone. The stimulus was presented as a circular stimulus tip of $1 \mathrm{~cm}$ in diameter, measured on a digital interval scale of $0.1 \mathrm{~N}$. Mechanical pressure was applied at a rate of $5 \mathrm{~N} / \mathrm{s}$ [24] and the mean PPT was calculated as the average of three consecutive measurements (Fig. 1c). The outcome is reported in Newtons.

\section{Statistical analysis}

Descriptive statistics are reported in mean and standard error or median and interquartile range according to the data distribution (normal or non-normal distribution, respectively). Statistical analysis was performed with a commercial software package Sigmaplot 12.0 for Windows (Systat software, Inc., Germany). The estimated sample size for the study was calculated using MHS as the main variable [14]. Taking into consideration a mean difference between sham and active intervention groups of $15 \%$, a standard deviation of $15 \%$, with a type I error $(\alpha)$ of 0.05 and a power of $80 \%$, a sample size of 10 subjects was calculated. However, as a contingency, the sample size was increased by four more subjects $(n=$ 14). The Gaussian distribution of the data was confirmed with the Shapiro-Walk test, and parametric statistical tests were adopted. A two-way repeated-measures ANOVA, with the "time" factor $(0 \mathrm{~min}, 15 \mathrm{~min}, 20 \mathrm{~min}$,

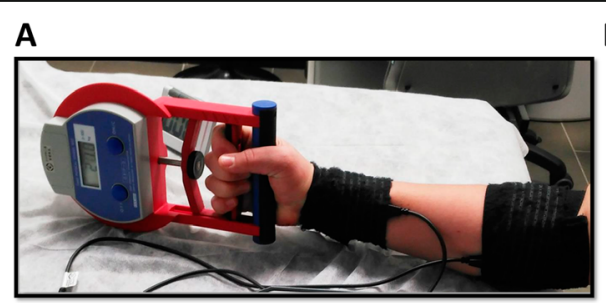

B

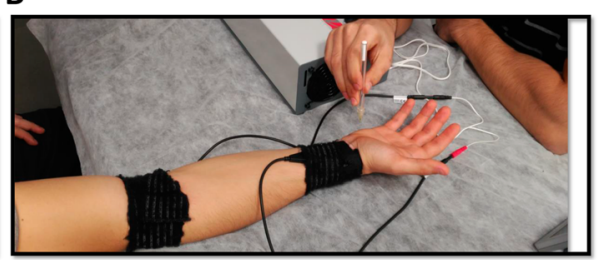

C

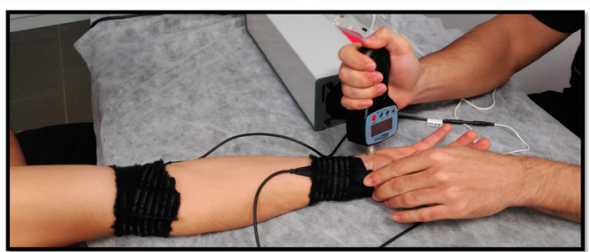

Fig. 1 Stimulus intervention, electrode placement and outcome measure assessment. a Maximal handgrip strength. b Mechanical detection threshold. c Pressure pain threshold 
$30 \mathrm{~min})$ and the "intervention" factor $(20 \mathrm{kHz}$ and sham stimulation), was performed to compare differences in MHS and PPT. Post-hoc analysis test was assessed with the Bonferroni test. Because MDT is a non-parametric measure, Friedman test was performed to analyse differences within time points $(0 \mathrm{~min}, 15 \mathrm{~min}, 20 \mathrm{~min}, 30$ min). U-Mann Whitney test was used to compare both interventions $(20 \mathrm{kHz}$ and sham stimulation). Post-hoc analysis test was assessed with the Tukey test. A $p$ value of $<0.05$ was considered statistically significant.

\section{Results}

Fourteen healthy volunteers completed the study 7 (50\%) males, mean age 33.3 years (SD 6.8) ranged between 23 and 44 years. No adverse effects were reported by any participant. The mean current intensity applied at the onset of the stimulus was $44.2 \mathrm{~mA}$ (SD 12.8), and the mean final intensity was $85.0 \mathrm{~mA}$ (SD 11.4; $p<$ $0.001)$.

\section{Maximal handgrip strength (MHS)}

Significant differences in the "time" factor $\left(\mathrm{F}_{(3,39)}=4.09\right.$; $p=0.013)$, the "intervention" factor $\left(\mathrm{F}_{(1,13)}=12.14 ; p=\right.$ $0.004)$ and the "time-intervention" interaction $\left(\mathrm{F}_{(3,39)}=\right.$ 4.09; $\mathrm{p}=0.013$ ) were detected. Specifically, $20 \mathrm{kHz}$ achieved a decrease in MHS at 15 min during the intervention (30.1 kgs, SE 2.8) when compared with baseline $(33.7 \mathrm{kgs}, \mathrm{SE} 2.8 ; p<0.001)$ and to the $10 \mathrm{~min}$ poststimulation time point $(32.7 \mathrm{kgs}$, SE $2.9 ; p<0.01)$. Sham stimulation did not show a significant change compared to baseline. A comparison of the both interventions revealed that the $20 \mathrm{kHz}$ stimulus produced a lower MHS (30.1 kgs, SE 2.8) when compared to sham stimulation (35.1 kgs, SE 3.4; $p<0.001$ ) during the stimulation at the 15 min test period. Immediately after the intervention at 20 min with $20 \mathrm{kHz}$ stimulation, MHS was statistically lower (31.8 kgs, SE 2.8) when compared to the same measure with sham stimulation $(34.2 \mathrm{kgs}$, SE $3.4 ; p=$ 0.03). (Fig. 2).

\section{Mechanical detection threshold (MDT)}

A statistically significant difference was found in the comparison of median values of $20 \mathrm{kHz}$ intervention among time points $(p<0.01)$, however the post-hoc test used did not reveal any significant difference. No statistical change in MDT was detected within sham stimulation. When the effect of active or sham stimulation were compared, the $20 \mathrm{kHz}$ stimulus revealed a slight increase in MDT $(0.25 \mathrm{mN} ; 0.25-2.00)$ at $15 \mathrm{~min}$ during the stimulation when compared to the sham stimulation $(0.25 \mathrm{mN}, 0.25-0.25 ; p=0.02)$ (Table 1$)$.

\section{Pressure pain threshold (PPT)}

No significant differences were found in any comparison for PPT, taking into consideration the "time" factor $\left(\mathrm{F}_{(3,39)}=0.54 ; p=0.66\right)$, the "intervention" factor $\left(\mathrm{F}_{(1,13)}=\right.$ $0.26 ; p=0.62)$, or the "intervention-time" intersection $\left(\mathrm{F}_{(3,39)}=0.84 ; p=0.48\right)($ Table 1$)$.

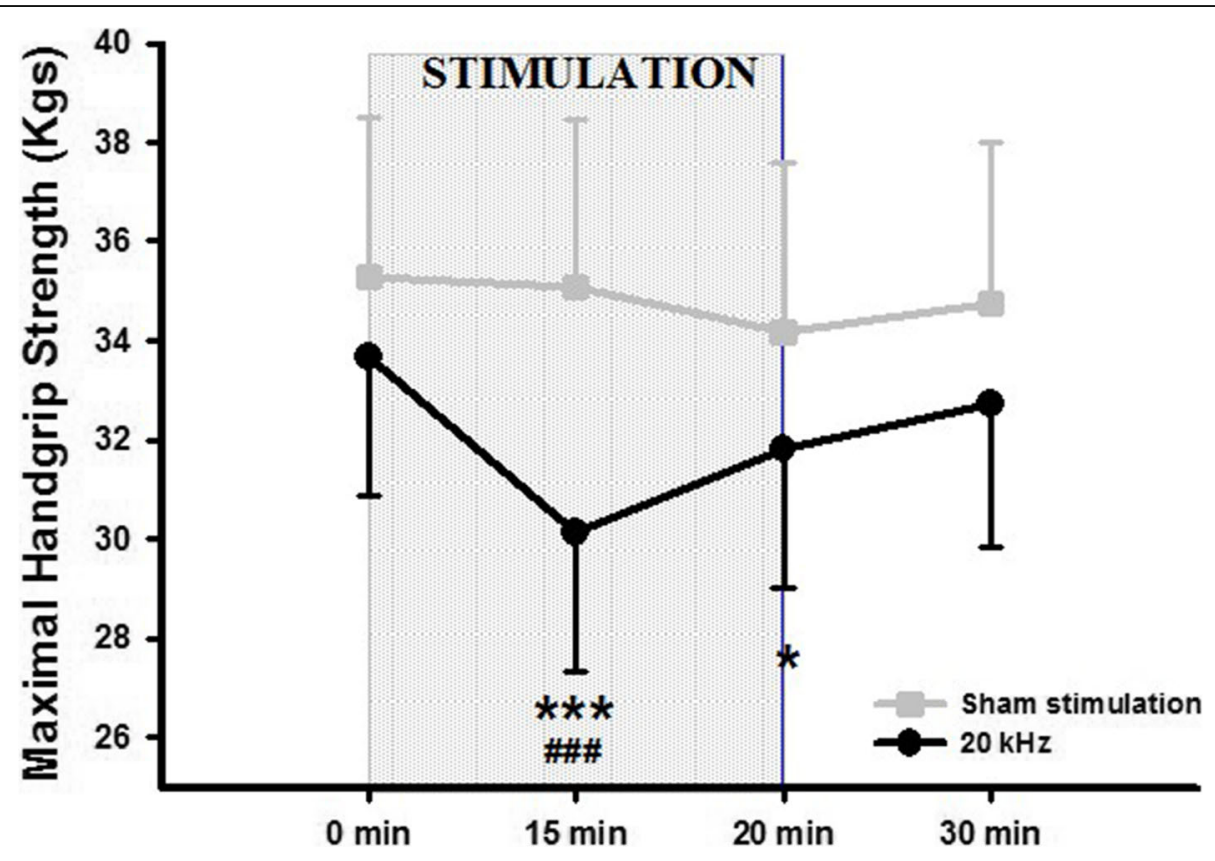

Fig. 2 HFAC stimulation effect on maximal handgrip strength. Sham stimulation (grey) and $20 \mathrm{kHz}$ (black). Data are represented as mean and standard error values. * Indicates value significantly different compared to sham stimulation $\left({ }^{* *} p<0.001 ;{ }^{*} p<0.05\right)$. \# Indicates significant difference from baseline and $30 \mathrm{~min}$ (\#\# $p<0.001$ ) 
Table 1 Changes in mechanical detection $(\mathrm{mN})$ and pressure pain threshold $(\mathrm{N} / \mathrm{cm} 2)$

\begin{tabular}{lllllll}
\hline & Intervention & $0 \mathrm{~min}$ & $15 \mathrm{~min}$ & $20 \mathrm{~min}$ & $30 \mathrm{~min}$ & Significance intragroup ${ }^{\text {(a) }}$ \\
\hline Mechanical Detection & $20 \mathrm{kHz}$ & $0.25(0.25-0.25)$ & $0.25(0.25-2.00)$ & $0.25(0.25-0.25)$ & $0.25(0.25-0.25)$ & $x^{2}: 12,75 ; p<0.01$ \\
Threshold & SHAM & $0.25(0.25-0.25)$ & $0.25(0.25-0.25)$ & $0.25(0.25-0.25)$ & $0.25(0.25-0.25)$ & $x^{2}: 0,00 ; p=1.00$ \\
& $\begin{array}{l}\text { Significance } \\
\text { intergroup (b) }\end{array}$ & $p=1.00$ & $p=0.02$ & $p=0.36$ & $p=1.00$ & \\
& $20 \mathrm{kHz}$ & $70.0(4.3)$ & $69.8(4.2)$ & $70.5(4.9)$ & $68.9(4.8)$ & "Time" factor: \\
$\begin{array}{l}\text { Pressure Pain } \\
\text { Threshold (c) }\end{array}$ & SHAM & $69.0(4.6)$ & $70.0(5.2)$ & $67.3(4.9)$ & $68.5(5.2)$ & $\begin{array}{l}\text { F: } 0.54 ; p=0.66 \\
\text { "Intervention" factor: F: } 0.26 ; p=0.62\end{array}$
\end{tabular}

Data of Mechanical Detection Threshold are expressed as median values (25-75\% inter-quartile range), and data of Pressure Pain Threshold as mean value (standard error). ${ }^{\text {(a) }}$ Friedman test. ${ }^{\text {(b) }}$ Mann-Whitney test. ${ }^{\text {(c) }}$ Two-way ANOVA repeated measures

\section{Discussion}

This is the first study showing the application of $20 \mathrm{kHz}$ stimulation in healthy participants over somatosensory and motor outcome measures. The main results of the present study showed that the application of transcutaneous HFAC at $20 \mathrm{kHz}$ over the median and ulnar nerves in healthy participants decreased maximal handgrip strength and slightly increased mechanical detection threshold when compared to sham stimulation. These results suggest that $20 \mathrm{kHz}$ stimulation could evoke a partial nerve conduction block, which has been previously demonstrated using a $10 \mathrm{kHz}$ stimulus [10, 14]. However, although the increase in MDT was statistically significant, the change was not determined to be clinically significant (Table 1 ). In addition, the pressure pain threshold did not change after the application of the stimulation. The difference of effect of $20 \mathrm{kHz}$ on specific sensory modalities may be due to specific effects on nerve fiber types as each has a different conduction block threshold, which in turn varies with the block frequency, observed in preclinical studies [25].

Previous studies have shown inhibited motor activity, Kim et al. [10] applied $10 \mathrm{kHz}$ HFAC stimulation over the median nerve and observed a decrease in finger muscle contraction of 40\%. Springer et al. [11] applied 7 $\mathrm{kHz}$ stimulation over the ulnar nerve and observed a decrease of $8 \%$ in maximal voluntary contraction and a $15 \%$ decrease in the myoelectrical activity of the abductor digiti minimi muscle, immediately after HFAC stimulation, indicating an effect on motor activity. The decrease in maximal handgrip strength with $20 \mathrm{kHz}$ HFAC stimulation observed in the present study (11\%) is in line with our previous study at $10 \mathrm{kHz}$ [14], where the reduction in MHS was of $14 \%$, at the same test times. The stimulation of proprioceptive fibers with the electrical stimulation may interfere with the accurate perception and performance of maximal voluntary muscle contraction under the test conditions, and may constitute one of the reasons why a reduction in muscle strength observed in this study.

Moreover, an increase in tactile and pressure pain threshold was also detected in previous studies at 5 and
$10 \mathrm{kHz}$, indicating decreased sensory function. Our previous study when HFAC stimulation was presented at 5 $\mathrm{kHz}$ to the radial nerve [7] the increase of MHS was of $17.2 \mathrm{mN}$. Kim et al. [10] with $10 \mathrm{kHz}$ observed an increase MDT to approximately twice that of the baseline condition during the stimulation. However, in our study with $20 \mathrm{kHz}$, HFAC stimulation was only able to block selectively motor activity assessed as MHS, with a slight increase on the threshold of $\mathrm{A} \beta$ fibers assessed by MDT, and without producing any significant differences on $A \delta$ sensory fibers, assessed as PPT. Because of this slight change on MDT, where the median value is the same, it could be supposed that the clinical effect could be not significant. This selective effect on motor activity could have interesting clinical implications, for example in people with the spasticity syndrome as the stimulation protocol would be able to specifically modulate the motor component without producing large changes in sensory pathways. This study also suggests that transcutaneous application of HFAC at $20 \mathrm{kHz}$ does not produce complete nerve conduction block in healthy volunteers, compared to the effect of direct nerve stimulation performed in preclinical studies using implanted electrodes [5, 26-28].

The discrepancy between the nerve conduction blockade in the animal and human studies could be explained by the greater distance between the stimulating electrode and the axon target during transcutaneous HFAC stimulation. However, the partial conduction block observed in our study, is in line with previous transcutaneous stimulation studies in human subjects [7, 10, 11, 14]. Of clinical relevance, our study showed that the effects of the stimulation were reverted immediately after the end of the stimulation, which is in consonance with previous preclinical studies [15]. In an "in vivo" study, the total time for recovery of normal function after stimulation lasted between $300 \mathrm{~ms}$ to $60 \mathrm{~s}$ [29]. Furthermore, an "in vitro" study reported that recovery of nerve function ranged between 4 to $10 \mathrm{~min}$, and also found that small diameter fibers recovered faster (C-fiber: $3 \mathrm{~min}$; $\mathrm{A} \delta$ fiber: $6 \mathrm{~min}$ ) [30]. These results suggest that stimulation protocols designed to block nerve conduction may have a 
preferential effect on motor fibers whose diameter are larger than smaller $\mathrm{A} \delta$ and $\mathrm{C}$ fibers, in addition to longer recovery time following nerve conduction block.

In this study, none of the participants reported adverse effects when HFAC was applied, and the modulation of motor and sensory nerve activity was quickly reversible after the stimulus, suggesting that the transcutaneous HFAC stimulation protocol was safe. Following several neuropathologies an aberrant increase in nerve activity may lead to specific symptoms of spasticity such as hypertonia, spasms, tremors, chronic pain could be reduced by the transcutaneous application of $20 \mathrm{kHz}$ HFAC by mediating a nerve conduction block, although the optimal stimulation frequency remains unknown. HFAC stimulation could have a great potential as a therapeutic tool for pathologies characterized by a hyperactivity of the nervous system, with the aim of inhibiting exaggerated nervous activity.

\section{Study limitations}

The main limitation of this study is that the sensory measures used are considered as quasi-objective, as their quantification relies on the perceived sensation and judgement of the participant. Neurophysiological measures such as motor and somatosensory evoked potentials may have more potential to objectively evaluate changes in nerve conduction during and after the stimulation. Another limitation is that the assessor was not blinded to the application of each intervention $(20 \mathrm{kHz}$ or sham stimulation). With regards to blinding procedures for subjects, the specific method used in this study has been used before $[7,20]$, although the success of the blinding technique was not formally tested. Finally, the present study was performed in healthy subjects and therefore conclusions about the applicability of the HFAC stimulation technique in patients should be made with caution until further studies are developed in participants with neurological disorders characterized by motor hyperactivity.

\section{Conclusions}

High frequency alternating current stimulation at a frequency of $20 \mathrm{kHz}$ applied over the ulnar and median nerves of healthy volunteers produces a decrease in muscle handgrip strength during the stimulation and a slight change in mechanical detection threshold, when compared to sham stimulation. HFAC at $20 \mathrm{kHz}$ has potential for clinical applications where a selective stimulation protocol is required to partially block alphamotoneurons without producing large changes in the sensory pathways.

\section{Abbreviations}

ANOVA: Analysis of variance; HFAC: High-frequency alternating current: MDT: Mechanical detection threshold; MHS: Maximal handgrip strength;
PPT: Pressure pain threshold; SD: Standard deviation; SE: Standard error; TENS: Transcutaneous electrical nerve stimulation

Authors' contributions

DSM and CSM contributed in participant recruitment, data collection and drafting manuscript. JAC, JGS and JT conceiving study concept, results interpretation and supervised the whole project. All authors read and approved the final manuscript.

\section{Funding}

This work was supported by the "Instituto de Salud Carlos III" (ref PI17/ 00581).

\section{Availability of data and materials}

The data collected in this study are available from the corresponding author on reasonable request.

\section{Ethics approval and consent to participate}

The local ethical committee of the "Complejo Hospitalario de Toledo" gave approval to the study and warranted its accordance with the Declaration of Helsinki (number 158; 2-11-2017). Written informed consent was obtained from all participants prior to participation.

\section{Consent for publication}

Not applicable.

\section{Competing interests}

Avendaño-Coy, Taylor and Gómez-Soriano have a patent of a device which evokes electrical current between $10 \mathrm{kHz}$ and $50 \mathrm{kHz}$, although this device has not used in this work. The remaining authors have no conflicts of interest.

\section{Author details \\ 'Sensorimotor Function Group, Hospital Nacional de Parapléjicos, 45071 Toledo, Spain. ${ }^{2}$ Toledo Physiotherapy Research Group (GIFTO), Faculty of Physiotherapy and Nursery, Castilla La Mancha University, 45071 Toledo, Spain. ${ }^{3}$ Department of Rehabilitation Sciences, KU Leuven - University of Leuven, 3000 Leuven, Belgium.}

Received: 27 June 2019 Accepted: 13 February 2020

Published online: 19 February 2020

\section{References}

1. Borsook D. Neurological diseases and pain. Brain. 2012;135:320-44.

2. Kilgore $\mathrm{KL}$, Bhadra N. Reversible nerve conduction block using kilohertz frequency alternating current. Neuromodulation. Apr 2014;17:242-54.

3. Kilgore $K L$, Bhadra N. Nerve conduction block utilising high-frequency alternating current. Med Biol Eng Comput. 2004:42:394-406.

4. Liu H, Roppolo JR, de Groat WC, Tai C. The role of slow potassium current in nerve conduction block induced by high-frequency biphasic electrical current. IEEE Trans Biomed Eng. 2009:56:137-46.

5. Tai C, Roppolo JR, de Groat WC. Block of external urethral sphincter contraction by high frequency electrical stimulation of pudendal nerve. J Urol. 2004;172:2069-72.

6. Camilleri M, Toouli J, Herrera MF, Kow L, Pantoja JP, Billington CJ, et al. Selection of electrical algorithms to treat obesity with intermittent vagal block using an implantable medical device. Surg Obes Relat Dis. 2009;5: 224-9; discussion 229-30.

7. Avendaño-Coy J, Gómez-Soriano J, Goicoechea-García C, Basco-López JA, Taylor J. Effect of Unmodulated 5-kHz alternating currents versus transcutaneous electrical nerve stimulation on mechanical and thermal pain, tactile threshold, and peripheral nerve conduction: a double-blind, placebo-controlled crossover trial. Arch Phys Med Rehabil. 2017;98:888-95.

8. Sarr MG, Billington CJ, Brancatisano R, Brancatisano A, Toouli J, Kow L, et al. The EMPOWER study: randomized, prospective, double-blind, multicenter trial of vagal blockade to induce weight loss in morbid obesity. Obes Surg. Nov 2012;22:1771-82.

9. Soin A, Shah NS, Fang Z-P. High-frequency electrical nerve block for postamputation pain: a pilot study. Neuromodulation. 2015;18:197-205. 
10. Kim Y, Cho HJ, Park HS. Technical development of transcutaneous electrical nerve inhibition using medium-frequency alternating current. J Neuroeng Rehabil. 2018;15:80.

11. Springer S, Kozol Z, Reznic Z. Ulnar nerve conduction block using surface kilohertz frequency alternating current: a feasibility study. Artif Organs. 2018; 42:841-6.

12. Shikora SA, Wolfe BM, Apovian CM, et al. Sustained Weight Loss with Vagal Nerve Blockade but Not with Sham: 18-Month Results of the ReCharge Trial. J Obes. 2015;2015:365604. https://doi.org/10.1155/2015/365604.

13. Rubinstein JT, Tyler RS, Johnson A, Brown CJ. Electrical suppression of tinnitus with high-rate pulse trains. Otol Neurotol. May 2003;24:478-85.

14. Serrano-Munoz D, Avendano-Coy J, Simon-Martinez C, Taylor J, GomezSoriano J. Effect of high-frequency alternating current transcutaneous stimulation over muscle strength: a controlled pilot study. J Neuroeng Rehabil. 2018:15:103.

15. Avendaño-Coy J, Serrano-Muñoz D, Taylor J, Goicoechea-García C, GómezSoriano J. Peripheral nerve conduction block by high-frequency alternating currents: a systematic review. IEEE Trans Neural Syst Rehabil Eng. 2018;26: $1131-40$.

16. Ackermann DM Jr, Ethier C, Foldes EL, Oby ER, Tyler D, Bauman M, et al. Electrical conduction block in large nerves: high-frequency current delivery in the nonhuman primate. Muscle Nerve. 2011:43:897-9.

17. Miles JD, Kilgore KL, Bhadra N, Lahowetz EA. Effects of ramped amplitude waveforms on the onset response of high-frequency mammalian nerve block. J Neural Eng. 2007:4:390-8.

18. Williamson RP, Andrews BJ. Localized electrical nerve blocking. IEEE Trans Biomed Eng. 2005;52:362-70

19. Zhang X, Roppolo JR, de Groat WC, Tai C. Simulation analysis of conduction block in myelinated axons induced by high-frequency biphasic rectangular pulses. IEEE Trans Biomed Eng. 2006;53:1433-6.

20. Claydon LS, Chesterton LS, Barlas P, Sim J. Alternating-frequency TENS effects on experimental pain in healthy human participants: a randomized placebo-controlled trial. Clin J Pain. 2013:29:533-9.

21. Aarskog R, Johnson MI, Demmink JH, Lofthus A, Iversen V, Lopes-Martins R, et al. Is mechanical pain threshold after transcutaneous electrical nerve stimulation (TENS) increased locally and unilaterally? A randomized placebocontrolled trial in healthy subjects. Physiother Res Int. 2007:12:251-63.

22. Serrano-Munoz D, Gomez-Soriano J, Bravo-Esteban E, Vazquez-Farinas M, Taylor J, Avendano-Coy J. Intensity matters: therapist-dependent dose of spinal transcutaneous electrical nerve stimulation. PLoS One. 2017;12: e0189734.

23. Ristic $D$, Spangenberg $P$, Ellrich J. Analgesic and antinociceptive effects of peripheral nerve neurostimulation in an advanced human experimental model. Eur J Pain. 2008:12:480-90

24. Chesterton LS, Barlas P, Foster NE, Lundeberg T, Wright CC, Baxter GD. Sensory stimulation (TENS): effects of parameter manipulation on mechanical pain thresholds in healthy human subjects. Pain. 2002;99:253-62.

25. Joseph L, Butera RJ. High-frequency stimulation selectively blocks different types of fibers in frog sciatic nerve. IEEE Trans Neural Syst Rehabil Eng. 2011; 19:550-7.

26. Ackermann DM, Foldes EL, Bhadra N, Kilgore KL. Conduction block of peripheral nerve using high-frequency alternating currents delivered through an intrafascicular electrode. Muscle Nerve. 2010;41:117-9.

27. Boger A, Bhadra N, Gustafson KJ. Different clinical electrodes achieve similar electrical nerve conduction block. J Neural Eng. 2013;10:056016.

28. Gerges M, Foldes EL, Ackermann DM, Bhadra N, Bhadra N, Kilgore KL. Frequency- and amplitude-transitioned waveforms mitigate the onset response in high-frequency nerve block. J Neural Eng. 2010;7:066003.

29. Dowden BR, Wark HA, Normann RA. Muscle-selective block using intrafascicular high-frequency alternating current. Muscle Nerve. 2010;42: 339-47.

30. Waataja JJ, Tweden KH, Christopher N, Honda CN. Effects of high-frequency alternating current on axonal conduction through the vagus nerve. J Neural Eng. 2011;8:056013.

\section{Publisher's Note}

Springer Nature remains neutral with regard to jurisdictional claims in published maps and institutional affiliations.

\section{Ready to submit your research? Choose BMC and benefit from:}

- fast, convenient online submission

- thorough peer review by experienced researchers in your field

- rapid publication on acceptance

- support for research data, including large and complex data types

- gold Open Access which fosters wider collaboration and increased citations

- maximum visibility for your research: over $100 \mathrm{M}$ website views per year

At $\mathrm{BMC}$, research is always in progress.

Learn more biomedcentral.com/submissions 